\title{
Changing epidemiology of non-cystic fibrosis bronchiectasis
}

\author{
Semiha Bahçeci ${ }^{1}$, Sait Karaman ${ }^{1}$, Hikmet Tekin Nacaroğlu ${ }^{1}$, Selçuk Yazıc1 ${ }^{2}$, Saniye Girit ${ }^{1}$, \\ Şule Ünsal-Karkıner ${ }^{1}$, Demet Can ${ }^{2}$ \\ ${ }^{1}$ Department of Pediatrics, Dr. Behçet Uz Pediatric Hospital, Izmir, and ${ }^{2}$ Department of Pediatrics, Balıkesir University \\ Faculty of Medicine Ballkesir, Turkey. E-mail: selcuk.yzci@gmail.com \\ Received: 5 October 2015, Revised 14 December 2015, Accepted: 13 April 2016
}

SUMMARY: Bahçeci S, Karaman S, Nacaroğlu HT, Yazıcı S, Girit S, ÜnsalKarkıner S, Can D. Changing epidemiology of non-cystic fibrosis bronchiectasis. Turk J Pediatr 2016; 58: 19-26.

Non-cystic fibrosis bronchiectasis again becomes a major health problem due to inappropriate antibiotic use and increasing frequency of protracted bacterial bronchitis. The aim was to determine the changes in etiology of bronchiectasis. Patients who admitted to Behçet Uz Children Hospital between 2005 and $2015(n=110)$ were retrospectively examined.

The etiology of bronchiectasis was detected as; primary ciliary dyskinesia $26.4 \%$, protracted bacterial bronchitis $22.8 \%$, primary immune deficiency $11.8 \%$, bronchiolitis obliterans $8.2 \%$, lung disease secondary to gastro-esophageal reflux $3.7 \%$, foreign body aspiration $2.7 \%$, tuberculosis $\% 2.7$, congenital malformation $1.8 \%$ and asthma $1.8 \%$, respectively. In $15.4 \%$ of cases, etiology was not identified clearly. $91 \%$ of the patients were medically treated.

In ten years, the frequency of asthma and tuberculosis in etiology had decreased but primary ciliary dyskinesia and primary immune deficiency had increased. Non-cystic fibrosis bronchiectasis can be followed up for a long time with medical treatment.

Key words: non-cystic fibrosis bronchiectasis, children, changing epidemiology.

Bronchiectasis (BE) is defined as a complex disorder characterized with enlargement of bronchi and thickening of bronchial walls by various reasons. The pathophysiology is not clearly understood ${ }^{1,2}$. Advances in sanitation, development in nutritional status, use of effective antibiotics for respiratory tract infections, success in childhood immunization (especially measles and pertussis) have led to a decrease in the disease ${ }^{1,3,4}$. In developed countries BE secondary to cystic fibrosis (CF) is common, but in less developed countries non-CF bronchiectasis is more frequent.

Research from developed countries report the most important etiologic factors for nonCF bronchiectasis development as infections (especially protracted bacterial bronchitis $(\mathrm{PBB})$ ), primary immune deficiency (PID), aspiration and primary ciliary dyskinesia $(\mathrm{PCD})^{3}$. The etiologic factors reported in our centers' etiology study 10 years ago $^{5}$ were as; lung infection $(34 \%)$, asthma (22\%), tuberculosis (TB) (20\%), chronic aspiration (8\%), PID (2\%) and PCD (2\%).

When high consanguineous marriage rate in our country is taken into account, genetic disorders like PID or PCD are expected to be higher than reported. Advances in complex diagnostic methods in the last 10 years brought a better understanding of non-CF bronchiectasis etiology. It can be suggested that, use of flexible bronchoscopy and use of flow cytometry for immunology in the last 10 years in our center may reveal a change in the rates of etiologic factors.

In the present study, the aim was to determine the probable etiologic changes and to evaluate the experience in follow-up of patients in the last ten years. We aimed to compare the etiological data of patients we followed up with the diagnosis of non-cystic fibrosis bronchiectasis between the years 2005-2015, with the data of the previous study published by our center in 2005 . 


\section{Material and Methods}

Patients, who were diagnosed as non-CF BE at the Pediatric Pulmonology Unit of Dr. Behçet Uz Children Hospital (İzmir, Turkey) between 2005 and 2015, were retrospectively evaluated. The diagnosis was confirmed by thorax High Resolution Computerized Tomography (HRCT) in the patients who had a persistent wet cough, and abnormal chest X-ray findings, which did not respond to antibiotic therapy and also maintained non-regressive symptoms for 2-4 weeks 6 .

\section{Inclusion criteria}

Presence of persistent lower respiratory tract complaints as chronic productive cough and repeating lung infections.

Detection of BE in HRCT by a radiologist.

Presence of tests for etiologic examination; complete blood count, peripheral smear, total eosinophil count, sputum culture, immunologic tests (pneumococcal vaccine response, IgG, IgA, IgM, IgE and IgG subgroups, lymphocyte panel, serum complement levels), sweat test, tuberculin skin test, $\alpha 1$-Antitripsin level, saccharin test, if indicated gastroesophageal scintigraphy, flexible bronchoscopy and bronchoalveolar lavage (BAL).

All of the mentioned tests, except for gastroesophageal scintigraphy and flexible fiberoptic bronchoscopy, were performed in each patient. Flexible fiberoptic bronchoscopy was performed in selected cases $(n=49$, $44.5 \%$ of the patients) especially in whom the etiology of BE could not be found by other diagnostic tests. Also rigid bronchoscopy was performed in 3 of 49 cases (cases with foreign body) who were performed flexible fiberoptic bronchoscopy. Sweat test was performed in all patients in order to exclude CF. In selected patients (atypical CF) genetic mutation analysis for $\mathrm{CF}$ was also performed.

The clinical and laboratory findings, detected bonchiectasis etiology, treatment course in follow-up period and prognosis were recorded for all patients. The data were compared with those of the previous study of our center which included the patients diagnosed as BE between 1995 and 20045 .

\section{Definitions of the etiologic causes PCD}

The BE patients were diagnosed as PCD 1) if they had dextrocardia or Kartagener Syndrome family history 2) if BE is accompanied by chronic sinusitis and if mucociliary movement in non-infected patients was not present or a positive saccharin test $>30$ minutes. When dextrocardia was detected in a patient or in any close relative who had clinical evidence of PCD (brother, mom, dad, aunt, uncle or cousins) the patient was considered as PCD. A sample of nasal scratch was obtained from each patient. Ciliary movements were evaluated by light microscopy in nasal epithelial samples. PCD was excluded in patients with ciliary movement.

Definitive diagnosis of PCD is achieved by light and electron microscopic examination of the ciliary functions and ultrastructural structures in brush biopsy samples taken from nasal or bronchial mucosa ${ }^{7,8}$. The frequency and pattern of the ciliary motion are examined under light microscopy ${ }^{7}$. In our study, the nasal mucosa samples taken with nasal scratch were examined under light microscopy by dripping saline on them. It was recorded whether there were cilia in the nasal epithelium and whether they were moving. Examples for this process were taken 4-6 weeks after any respiratory tract infection due to the reason that they can cause secondary ciliary dyskinesia. If ciliar movement was observed in light microscopy, the ciliar activity was deemed as normal. When cilia were absent or still the ciliar activity was considered as suspicious. If there was suspicious ciliary activity and it was not possible to show the ciliary transport by saccharin test, then it was accepted as mucociliary dysfunction.

Evaluation of mucociliary movement and also the saccharin test were both performed in compliant patients. Tasting saccharin in less than 30 minutes was considered as normal. Both tests were performed at least 2 times during the infection-free period.

\section{Asthma and reactive airway disease (asthma- like symptoms)}

The asthma diagnosis was based on the patients' symptoms and medical history, and was supported with laboratory findings. Both the modified Asthma Predictive Index (mAPI) and Global Initiative for Asthma (GINA) report 2014 were used ${ }^{9,10}$. The patients, who had recurrent episodes $(\geq 4)$ of wheezing, 
that responded to inhaled steroids and/or bronchodilators taken within 2-4 weeks, were diagnosed as reactive airway disease.

\section{Protracted bacterial bronchitis}

The diagnosis was based on the presence of a chronic, wet cough, having the appropriate response to the antibiotic therapy and resolution of the cough within 2-4 weeks. No other alternative causes of this specific cough were found ${ }^{11}$. The flexible bronchoscopy was also performed to exclude any other causes and to detect specific bacterial etiologies and neutrophilia in the bronchoalveolar lavage fluid (BALF).

\section{Gastroesophageal reflux disease (GERD)}

The patients who responded to the treatment of a proton pump inhibitor within 2 to 4 weeks and who were diagnosed by a pediatric gastroenterelogist via gastroesophageal scintigraphy and a gastroduodenoscopy were involved in this group.

\section{Tuberculosis}

The diagnosis was usually based on persistent symptoms unresponsive to antibiotherapy, chest radiography findings, tuberculin skin test positivity, positive microbiologic tests, and history of contact with people having TB disease. The patient was considered as TB if there was positivity in microbiological tests or tuberculin skin test, history of contact with people having TB disease, persistent symptoms unresponsive to antibiotherapy and chest radiography findings.

\section{Bronchiolitis obliterans (BO)}

The diagnosis was based on the following criteria: a) patients with no other respiratory disease from birth to the onset of the acute disease such as bronchiolitis or severe pneumonia, b) patients with persistent obstructive respiratory symptoms for at least 60 days after the initial illness, c) patients with HRCT findings in a mosaic pattern, vascular attenuation and expiratory air trapping and d) excluding other diagnoses ${ }^{12}$.

\section{Massive hemoptysis}

Volumes of 100 to $1000 \mathrm{ml}$ of blood have been described as indicative of hemoptysis, but no specific volume has been universally accepted ${ }^{13}$.

\section{Medical treatment}

All patients were followed up with medical treatment including prompt antibiotic use in exacerbations, bronchodilators and physiotherapy. Inhaled steroids were given to patients who had symptoms suggestive of bronchial hyperreactivity and positive bronchodilator responses.

\section{Surgical Treatment}

Patients who had recurrent/persistent respiratory symptoms despite intensive medical treatment required surgery.

\section{Results}

A hundred and ten patients were diagnosed as non-CF BE in 10 years period. $50.9 \%$ were male. Average age of patients was $167 \pm 39$ months, average age at the diagnosis was $102 \pm 42$ months and average follow up duration was $65 \pm 41$ months.

Etiology of BE was detected respectively as; PCD $26.4 \%(n=29)$, persistent bacterial bronchitis $22.8 \%(n=25)$, PID $11.8 \%(n=13)$, BO $8.2 \%(n=9)$, lung disease secondary to gastro-esophageal reflux $3.7 \%(n=4)$, foreign body aspiration $2.7 \%(\mathrm{n}=3)$, TB $2.7 \%(\mathrm{n}=3)$, congenital airway anomaly (one pulmonary sequestration and one idiopathic congenital BE) $1.8 \%(n=2)$ and asthma $1.8 \%(n=2)$. In $15.4 \%(n=17)$ of cases, etiology was not identified clearly and those were diagnosed as idiopathic BE. When compared with the previous study, it was clear that, the etiologic factors have changed in ten years and the frequency of asthma and tuberculosis in etiology had decreased (Table I).

In $49 \%$ of the cases the lower lobes were affected, in $31.6 \%$ of the cases also BE in right medial lobe and/or lingula accompanied the lower lobe involvement.

Surgical approach was mandatory for 10 (9\%) patients and the rest of cases were medically treated. Lobectomy was performed to localized BE with history of frequent exacerbations or hemoptysis. No patients underwent pneumonectomy. Etiology of patients who underwent surgery $(n=10) ; 2$ cases with congenital airway anomaly, 7 patients with postinfectious BE (recurrent exacerbations and severe infection) and one patient with Hodgkin's lymphoma (recurrent exacerbations and severe 
Table I. Comparison of Two Studies From Our Center: Changes in Etiologic Factors For Non-CF Bronchiectasis by Time

\begin{tabular}{lccccc}
\hline & \multicolumn{2}{c}{$2005-2015$} & & \multicolumn{2}{c}{$1995-2005$} \\
\cline { 2 - 3 } \cline { 5 - 6 } & $\mathrm{n}$ & $\%$ & & $\mathrm{n}$ & $\%$ \\
\hline Primary ciliary dyskinesia & 29 & 26.4 & & 1 & 2 \\
Infection & $26^{*}$ & 23.7 & & 18 & 36 \\
Unknown etiology & 17 & 15.4 & & 0 & 0 \\
Primary immune deficiency & 13 & 11.8 & & 1 & 2 \\
Bronchiolitis obliterans & 9 & 8.2 & & 0 & 0 \\
Aspiration/foreign body & 7 & 6.4 & & 5 & 10 \\
Tuberculosis & 3 & 2.7 & & 10 & 20 \\
Congenital malformation & 2 & 1.8 & & 0 & 0 \\
Secondary immune deficiency & 2 & 1.8 & & 0 & 0 \\
Asthma & 2 & 1.8 & & 11 & 22 \\
$\alpha 1$-antitrypsin deficiency & 0 & 0 & 1 & 2 \\
Total & 110 & 100 & 47 & $94^{* *}$ \\
\hline
\end{tabular}

*Protracted bacterial bronchitis (22.8\%)

** Missing three cases; CF (6\%)

infection). The most frequent complications were acute exacerbation (90\%) and hemoptysis (2.7\%). Hemoptysis has been shown in only 3 patients [2 Kartagener Syndrome, one Bruton disease (PID)].Hemoptysis was observed due to infection and has improved with treatment of infections. Massive hemoptysis was not detected. All included patients are still followed up in our clinic and no death has occurred up to date.

The most frequent etiologic factor was PCD $(n=29)$. Fifty two percent of PCD cases $(15 / 29)$ were Kartagener Syndrome and $48 \%$ of patients were without situs inversus. In patients without situs inversus, the diagnoses have been achieved by presence of bronchiectasis, presence of family history for Kartagener's syndrome, lack of mucociliary activity, a saccharin test $>30$ minutes in cooperative cases and by ruling out other causes and etiological reasons.

Diagnosis in 5 of the patients without situs anomaly $(n=14)$ was supported by presence of Kartagener Syndrome in brothers or sisters. In the remaining 9, diagnosis was supported by presence of chronic sinusitis, lack of mucociliary movement, a positive saccharin test $>30$ minutes and exclusion of other possible causes.

The second most frequent cause of non-CF BE was infection. Twenty five of infection cases were PBB (22.8\%), 3 were TB $(2.7 \%)$ and one was $(0.9 \%)$ cyst hydatid infection (Table I). Bacterial growth in BAL of PBB diagnosed cases; Haemophilus influenzae type $B$ was detected in 9 of 26 cases (36\%), Streptococcus pneumoniae in $6(24 \%)$ and Staphylococcus aureus in one case (4\%). No growth was found in the remaining PBB cases. In patients diagnosed as nonPBB that BAL could be applied: Pseudomonas aeruginosa was detected in 6 patients, Klebsiella pneumoniae in one case and Candida albicans in one case.

Primary immune deficiency $(n=13)$ was the third most common etiologic factor (11.8\%). The PID patients are shown in details in Table II.

Aspiration was the etiologic factor in 7 patients $(6.4 \%)$. Four of them were gasteroesophageal reflux patients and 3 were due to foreign body aspiration. One of the four cases received the diagnosis of gastroesophageal reflux by showing the lipid laden macrophages in BAL and other 3 patients by gastroesophageal scintigraphy, gastroduodenoscopy performed by gastroenterologists and by evaluating the response to treatment.

Both cases of secondary immune deficiency $(1.8 \%)$ were Hodgkin Lymphoma patients. Only two $(1.8 \%)$ cases had congenital malformations (one pulmonary sequestration and one idiopathic congenital $\mathrm{BE}$ ). No reason 
was detected by analyses focusing on the etiology in the case that had recurrent lung infections since the age of six months and bilateral bronchiectasis in thorax tomography. Case was operated at the age 3. Since there was no finding in biopsy material, the patient was considered to have $\mathrm{BE}$ due to idiopathic congenital etiology.

The study clearly revealed that, PID and PCD increased in the etiology of non-BF bronchiectasis in ten years period (Table I).

\section{Discussion}

Effective treatment of infectious diseases and advances in diagnostic methods led to changes in the etiology of non-CF BE. Our current study, performed at the same center with 10 years interval, revealed changes in the etiology of non-CF BE. By the time, the frequencies of asthma and tuberculosis decreased but frequencies of PID and PCD increased.

Bronchiectasis is still an important health care problem in developing countries ${ }^{1,3,4}$. In children with BE usually slowly persisting chronic respiratory symptoms are present ${ }^{14,15}$. In cases with chronic productive cough and relapsing lower airway infections clinician should be suspicious for BE. Additionally, BE should be kept in mind in patients with rhinosinusitis, malaise, hemoptysis and treatment resistant asthma $2-4,12,14-16$. Consistent with previous studies, productive cough was the most common symptom in our patients ${ }^{4,15}$.

Productive cough is the most common symptom and may start several years before the diagnosis-4,12,14-16. Recent research in children revealed that the diagnosis is usually within 3-3.6 years after the onset of symptoms. The age of diagnosis in studies from our country is about eight; as similar to our study ${ }^{16-18}$.
According to the non-CF BE guideline of British Thoracic Society, medical history, history of viral infection or pneumonia in childhood, presence of gastric aspiration, asthma, infertility or connective tissue disorder should be asked when evaluating the patient. Family history should be detailed for genetic disorders like $\mathrm{PCD}^{6,14}$.

Brower KS et al. ${ }^{3}$ evaluated 989 patients, who were diagnosed between 1997 and 2002, for etiological reasons. Data from 12 studies (2 from our country) were evaluated in this metaanalysis. This meta-analysis showed that, the most frequent etiologic reasons are infections, PID, aspiration (foreign body aspiration and repeating aspirations in neuro-motor retardant patients) and PCD (Table III). When examined in details, severe bacterial or viral pneumonia in infection group and B cell anomalies in immune-deficiency group are reported as the most frequent reasons ${ }^{3}$. The order of etiologic reasons in our patients is as; 1) PCD, 2) PID, 3) $\mathrm{PBB}, 4)$ Congenital respiratory tract disease and 5) Asthma.

Diagnosis of PCD is completed by presence of dextrocardia and presence of symptoms in brothers or sisters. Eight of our patients are diagnosed as PCD by saccharine test or absence of mucociliary movement in non-infected patients. A sample of nasal scratch was obtained from each patient. Ciliary movements were evaluated by light microscopy in nasal epithelial samples. PCD was excluded in patients with ciliary movement. The nasal saccharin test and evaluating the ciliary movements with light microscopy are not valuable in the diagnosis. Despite the fact that, ciliary structural abnormalities identified by electron microscopy of nasal epithelial biopsies have been the gold standard for diagnosing PCD, we were unable to perform electron microscopic

Table II. Primary Immune Deficiency Disorders in Non-CF Bronchiectasis Patients $(n=13)$

\begin{tabular}{lcc}
\hline & $\mathrm{n}$ & $\%$ \\
\hline B cell disease & 8 & 61.5 \\
Bruton disease & 2 & 15.4 \\
Common variable immune deficiency (cvid) & 3 & 23.1 \\
Iga deficiency & 2 & 15.4 \\
Igg subclass deficiency & 1 & 7.7 \\
Combined immune deficiency & 5 & 38.5 \\
Hyperimmunoglobulin E syndrome & 2 & 15.4 \\
Ataxia telangiectasia & 2 & 15.4 \\
Nijmegen Breakage syndrome & 1 & 7.7 \\
\hline
\end{tabular}


examination in our center. Another limitation of our study was the inability to use nasal nitric oxide (NO) as scanning test.

The frequency of PCD in BE etiology has dramatically increased when compared to our previous study (from $2 \%$ to $26.4 \%$ ). This result may be related with the foundation of Chest Diseases Unit in our center and changes in the centers' policy that recommend investigating PCD in all bronchiectasis patients who has dextrocardia or history of consanguineous marriage. Even in the group with any situs anomaly or consanguineous marriage history, there may be missing PCD patients due to the fact that complicated diagnostic tests are not performed in the idiopathic group.

Tuberculosis was diagnosed in 3 of $\mathrm{BE}$ cases that were secondary to infection. One of the remaining cases was diagnosed as cyst hydatid and 25 of them as PBB. In two of the patients, the diagnosis of tuberculosis was achieved by presence of suspicious clinical findings as well as tuberculin skin test (TST) positivity and history of contact. In the other patient bacillus was positive in sputum. In the case with cyst hydatid, it has been interpreted by the radiologist that the compression of a large cyst might have caused $\mathrm{BE}$ and bronchomalacia. We did not detect any BE case secondary to vaccine preventable diseases like measles or pertussis.
The study of Brower KS et $a .^{3}$ revealed that $11 \%$ of the non-CF BE patients secondary to infection $(n=108)$ were diagnosed as tuberculosis. $2.7 \%$ of our cases were diagnosed as tuberculosis. However, in our previous study 5 TB was the third etiological reason with a rate of $20 \%$. By advances in diagnostic methods the underlying cause was detected as PID in 3 patients formerly diagnosed as only TB.

The study of Brower KS et al. ${ }^{3}$ revealed that $17 \%$ of non-CF BE patients were PID patients, the majority of which was antibody deficiency disorders. Karadağ et al. ${ }^{16}$ reported the rate of PID in non-CF BE etiology as $15.3 \%$, Doğru et al. ${ }^{18}$ as $11.8 \%$, Karakoç et al. ${ }^{17}$ as $9.1 \%$, Zaid et al. ${ }^{19}$ as $22 \%$ and $\mathrm{Li}$ et al. ${ }^{20}$ as $29.4 \%$. PID was detected in $11.8 \%$ of our patients. $61.5 \%$ of these cases were antibody deficiency disorders (Table II).

The non-CF BE guideline of British Thoracic Society recommends to investigate all $\mathrm{BE}$ patients for immune deficiency, especially for antibody deficiency disorders and also recommends cooperation of pulmonologists and immunologists in the diagnosis, treatment and follow up of the disease. Consistent with the guideline, our rate of PID has increased with our increased cooperation with immunologists and with advances in our immunology laboratory tests. Similarly, close cooperation with radiologists and availability of HRCT use

Table III. Comparison of Studies About Etiology of Non-CF Bronchiectasis With our Study

\begin{tabular}{|c|c|c|c|c|c|c|c|c|c|c|}
\hline & \multicolumn{2}{|c|}{$\begin{array}{c}\text { Brower } \\
\text { KS et al. } \\
(2014)\end{array}$} & \multicolumn{2}{|c|}{$\begin{array}{l}\text { Karakoç et } \\
\text { al. (2009) }\end{array}$} & \multicolumn{2}{|c|}{$\begin{array}{l}\text { Karadağ et } \\
\text { al. (2005) }\end{array}$} & \multicolumn{2}{|c|}{$\begin{array}{l}\text { Doğru et al. } \\
\qquad(2005)\end{array}$} & \multicolumn{2}{|c|}{$\begin{array}{l}\text { Our study } \\
(2015)\end{array}$} \\
\hline & $\mathrm{n}$ & $\%$ & $\mathrm{n}$ & $\%$ & $\mathrm{n}$ & $\%$ & $\mathrm{n}$ & $\%$ & $\mathrm{n}$ & $\%$ \\
\hline Infection & 174 & 19 & 9 & 41 & 33 & 29.7 & $33^{* *}$ & 16.1 & $29 * * *$ & 26.4 \\
\hline Primary immunodeficiency & 158 & 17 & 2 & 9.1 & 17 & 15.3 & 11 & 5.4 & 13 & 11.8 \\
\hline Aspiration/foreign body & 91 & 10 & 0 & 0 & 4 & 3.6 & 7 & 3.4 & 7 & 6.4 \\
\hline Primary ciliary dyskinesia & 66 & 7 & 1 & 4.5 & 7 & 6.3 & 24 & 11.8 & 29 & 26.4 \\
\hline Congenital malformation & 34 & 4 & 0 & 0 & 3 & 2.7 & 1 & 0.5 & 2 & 1.8 \\
\hline $\begin{array}{l}\text { Secondary immune } \\
\text { deficiency }\end{array}$ & 29 & 3 & 0 & 0 & 0 & 0 & 0 & 0 & 2 & 1.8 \\
\hline Asthma & 16 & 2 & 4 & 18.2 & 5 & 4.5 & 24 & 11.8 & 2 & 1.8 \\
\hline Bronchiolitis obliterans & 12 & 1 & 0 & 0 & 0 & 0 & 0 & 0 & 9 & 8.2 \\
\hline Unknown etiology & 308 & 34 & 6 & 27.2 & 42 & 37.8 & 100 & 49 & 17 & 15.4 \\
\hline Other & 0 & 0 & 0 & 0 & 0 & 0 & $4^{*}$ & 2 & 0 & 0 \\
\hline
\end{tabular}

* 2 radiotherapy and chemotherapy (1\%), 1 sulphur inhalation $(0.5 \%), 1$ yellow nail syndrome $(0.5 \%)$

** 10 of 33 infections are Tuberculosis (4.9\%)

*** 25 protracted bacterial bronchitis $(22.8 \%), 3$ Tuberculosis $(2.7 \%)$ and a cyst hydatid $(0.9 \%)$ case 
in our clinic may be beneficial in diagnosing $\mathrm{BO}$ in $8.2 \%$ of our patients.

Karadağ et al. ${ }^{16}$ did not report any case of BO in 111 BE patients, Doğru et al. ${ }^{18}$ in 204 patients and also Karakoç et al. ${ }^{17}$ did not in $22 \mathrm{BE}$ patients. But $\mathrm{BO}$ rate in the study of Eastham et al. ${ }^{21}$ is $8.6 \%$ and is similar to our rate.

Aspiration was present in $6.4 \%(n=7)$ of our cases which involved cases secondary to GER and foreign body aspiration. There was no aspiration case due to neurologic reasons. Zaid et al. ${ }^{19}$ reported rate of BE secondary to aspiration as $22 \%$ and $\mathrm{Li}$ et al. ${ }^{20}$ as $18 \%$. In the studies from our country, Karadağ et al. ${ }^{16}$ reported the rate as $3.6 \%$ and Doğru et al. ${ }^{18}$ as $3.4 \%$ but Karakoç et al. ${ }^{17}$ reported no case of $\mathrm{BE}$ secondary to aspiration. In our previous study our rate was higher ${ }^{5}$. We conclude that, the decrease is related to the use of flexible bronchoscopy by which foreign bodies are rapidly removed before $\mathrm{BE}$ development.

In $1.8 \%$ of our cases $\mathrm{BE}$ secondary to asthma was detected. The BE rate secondary to asthma was reported as $31.8 \%$ by Gillard et al.22, as $18.2 \%$ by Karakoçet et al. ${ }^{17}$, as $11.8 \%$ by Doğru et al. ${ }^{18}$, and as $4.5 \%$ by Karadağ et al. ${ }^{16}$ in their cases. What may be the reason for the decrease of asthma rates in the present study? We conclude that the BE patients who has very frequent bronchospasms may be over-diagnosed as asthma. But in the long term follow up period, evaluation of acute exacerbations and pulmonary function tests may result in changes in the diagnosis. Thus, some patients of our previous study is re-classified as idiopathic cases in the present study due to the detection of fixed airway obstruction in pulmonary function tests in the follow up period. Only 2 cases with severe asthma who were not consistent to treatment remained in the asthma group.

The rate of congenital malformations in $\mathrm{BE}$ etiology differs between $1 \%$ and $13.6 \%^{14-20}$. $1.8 \%$ of our cases had congenital malformations (one pulmonary sequestration and one idiopathic congenital BE) (Table I). Doğru et al. ${ }^{18}$ reported only one congenital malformation (chest deformity) in 204 patients (0.5\%).

Localization of BE is determined by $\mathrm{HRCT}^{2,6}$. Opposite to the upper lobe involvement in CF patients, lower lobe involvement is more frequent in non-CF BE. Lower lobe BE was present in $49 \%$ of our patients.

Despite advances in diagnose and treatment of $\mathrm{BE}$, delays in diagnose are still very frequent and also a large amount of cases are not diagnosed. In approximately $55 \%$ of BE patients the etiology is not clear ${ }^{16-23}$. We were unable to detect the etiology in $15.4 \%(n=17)$ of our cases.

In conclusion, advances in diagnostic methods and impairment in the awareness of disease among clinicians has changed the distribution of etiological factors for BE. In the last ten years period PID and PCD have increased in the etiology. The increase of genetic disorders in the etiology of $\mathrm{BE}$ is consistent with the high consanguineous marriage rate in our country. Our study is also an important model that, despite $\mathrm{BE}$ is a persistent airway disorder and surgical approach is very common, nonCF BE patients can be followed up for years without any complications with proper medical treatment.

\section{REFERENCES}

1. Notarangelo LD, Plebani A, Mazzolari E, et al. Genetic causes of bronchiectasis: primary immune deficiencies and the lung. Respiration 2007; 74: 264-275.

2. King PT. The pathophysiology of bronchiectasis. Intern J Chron Obstruct Pulmon Dis 2009; 4: 411-419.

3. Brower KS, Del Vecchio MT, Aronoff SC. The etiologies of non-CF bronchiectasis in childhood: a systematic review of 989 subjects. BMC Pediatr 2014; 14: 229.

4. Banjar HH. A review of 151 cases of pediatric noncystic fibrosis bronchiectasis in a tertiary care center. Ann Thorac Med 2007; 2: 3-8.

5. Gerçek H, Can D, Altınöz S, et al. Bronşiektazili 50 pediatrik olgunun değerlendirilmesi. Toraks Dergisi 2006; 7: 101-104.

6. Pasteur MC, Bilton D, Hill AT. British Thoracic Society Bronchiectasis non-CF Guideline Group. British Thoracic Society guideline for non-CF bronchiectasis. Thorax 2010; 65: 1-58.

7. Hogg C. Primary ciliary dyskinesia: when to suspect the diagnosis and how to confirm it. Paediatr Respir Rev 2009; 10: 44-50.

8. Barbato A, Frischer T, Kuehni CE, et al. Primary ciliary dyskinesia: a consensus statement on diagnostic and treatment approaches in children. Eur Respir J 2009; 34: 1264-1276.

9. Guilbert TW, Morgan WJ, Krawiec M, et al. Prevention of Early Asthma in Kids Study, Childhood Asthma Research and Education Network. The Prevention of Early Asthma in Kids study: design, rationale and methods for the Childhood Asthma Research and 
Education network. Control Clin Trials 2004; 25: 286-310.

10. Global Initiative for Asthma. GINA report, global strategy for asthma management and prevention. Revised 2014. Available at: www.ginasthma.org.

11. Chang $A B$, Robertson $C F$, van Asperen PP, et al. A cough algorithm for chronic cough in children: a multicenter, randomized controlled study. Pediatrics 2013; 131: e1576-1583.

12. Champs NS, Lasmar LM, Camargos PA, et al. Postinfectious bronchiolitis obliterans in children. J Pediatr 2011; 87: 187-198

13. Larici AR, Franchi P, Occhipinti M, et al. Diagnosis and management of hemoptysis. Diagn Interv Radiol 2014; 20: 299-309.

14. McShane PJ, Naureckas ET, Tino G, et al. Non-cystic fibrosis bronchiectasis. Am J Respir Crit Care Med 2013; 188: 647-656.

15. McDonnell MJ, Ward C, Lordan JL, et al. Non-cystic fibrosis bronchiectasis. QJM 2013; 106: 709-715.

16. Karadag B, Karakoc F, Ersu R, et al. Non-cystic-fibrosis bronchiectasis in children: a persisting problem in developing countries. Respiration 2005; 72: 233-238.

17. Karakoc GB, Inal A, Yilmaz M, et al. Exhaled breath condensate MMP-9 levels in children with bronchiectasis. Pediatr Pulmonol 2009; 44: 1010-1016.

18. Doğru D, Nik-Ain A, Kiper N, et al. Bronchiectasis: the consequence of late diagnosis in chronic respiratory symptoms. J Trop Pediatr 2005; 51: 362-365.

19. Zaid AA, Elnazir B, Greally P. A decade of non-cystic fibrosis bronchiectasis 1996-2006. Ir Med J 2010; 103: 77-79.

20. Li AM, Sonnappa S, Lex C, et al. Non-CF bronchiectasis: does knowing the aetiology lead to changes in management? EurRespir J 2005; 26: 8-14.

21. Eastham KM, Fall AJ, Mitchell L, et al. The need to redefine non-cystic fibrosis bronchiectasis in childhood. Thorax 2004; 59: 324-327.

22. Gaillard EA, Carty H, Heaf D, et al. Reversible bronchial dilatation in children: comparison of serial high-resolution computer tomography scans of the lungs. Eur J Radiol 2003; 47: 215-220.

23. Gao YH, Guan WJ, Xu G, et al. Macrolide therapy in adults and children with non-cystic fibrosis bronchiectasis: a systematic review and meta-analysis. PLoS One. 2014; 9:e90047. 